\title{
Die Wohnungsfrage abseits der Metropolen: Wohnen in Salzburg zwischen touristischer Nachfrage und Finanzanlagen
}

\author{
Andreas Van-Hametner ${ }^{1}$, Christian Smigiel ${ }^{1}$, Karolin Kautzschmann ${ }^{2}$, and Christian Zeller ${ }^{1}$ \\ ${ }^{1}$ Fachbereich Geographie und Geologie, Universität Salzburg, Salzburg, Österreich \\ ${ }^{2}$ Institut für Wirtschafts- und Kulturgeographie, Leibniz-Universität Hannover, Hannover, Deutschland
}

Correspondence: Andreas Van-Hametner (andreas.van-hametner@sbg.ac.at)

Received: 27 September 2018 - Revised: 27 March 2019 - Accepted: 27 May 2019 - Published: 28 June 2019

\begin{abstract}
Kurzfassung. The housing question is a topic of increasing concern in a number of European cities. Rising housing costs burden many households. Since the outbreak of the global economic crisis in 2008, housing has increasingly come under spotlight of investors. Institutional and private actors invest in real estate not only in metropolitan areas but also in middle and small-sized towns. Tourism-induced demand increases the pressure on housing markets particularly in tourist-dominated cities. The manifestation of these processes varies on a small scale and is influenced by regional-local planning and politics. Based on the example of the city of Salzburg, the article shows that the housing question manifests itself strongly aside metropolises as well. Financial investments by private and institutional actors in real estate and different forms of tourism demand exacerbate the housing shortage. By linking the discussions about the effects of (mass-)tourism and financial investments on regional housing markets, this paper provides additional insights. The additional demand for (residential) space by tourism is used to increase or secure the return on investments in real estate. Financial investments in housing offer additional (problematic) potential for tourist use, such as short-term rentals. Owners benefit from both processes. Due to the prevalent power relations, planning and politics have little to counter the current challenges.
\end{abstract}

\section{Einleitung: die Wohnungsfrage in Salzburg}

Die Wohnkosten sind in vielen europäischen Städten ins Zentrum des öffentlichen Interesses geraten: Die ,Wohnungsfrage" ist wieder allgegenwärtig. Starke Preissteigerungen belasten viele Haushalte, im Besonderen aber Niedrigverdiener_innen. Sie sind gezwungen immer größere Anteile ihres Einkommens für Wohnen aufzuwenden (Schönig et al., 2017:12; Lebuhn et al., 2017). Die Wohnungsfrage stellt sich aber nicht nur in rein finanzieller Hinsicht, sondern ist immer eine gesellschaftliche und politische Frage. In konzeptioneller Hinsicht ist die Wohnungsfrage somit stets mit spezifischen Macht- und Kräfteverhältnissen verbunden, die es freizulegen gilt, will man einerseits die lokale und regionale Bedeutung der Wohnungsfrage verstehen und andererseits eine Suche nach Möglichkeiten des Gestaltens anstoBen (vgl. Zeller et al., 2018). Dies umfasst konkret die Rolle der politischen Institutionen auf nationaler und lokaler Ebe- ne, die Stadt- und Raumplanung, welche die Diskrepanz von Wohnen zwischen Grundbedürfnis und Ware mitbestimmen.

Die Zuspitzung der Probleme am Wohnungsmarkt zeigte sich zuletzt seit der Subprime-Krise weltweit. Diese „Wohnungsfrage" manifestiert sich jedoch in jeder Stadtregion auf ihre eigene spezifische Weise (Marcuse and Madden, 2016). Institutionelle und wirtschaftspolitische Rahmenbedingungen auf nationaler Ebene sind demnach zwar wichtig und einflussnehmend. Die konkreten Ausdifferenzierungen der Wohnungsfrage und Entwicklungen auf den Märkten für Eigentums- und Mietwohnungen divergieren jedoch bei kleinräumiger Betrachtung wesentlich, weshalb es wichtig ist, die regional-lokale Ebene genauer zu betrachten (vgl. Lebuhn et al., 2017).

Deshalb konzentrieren wir uns in diesem Beitrag auf einen konkreten regionalen Kontext - die Stadt Salzburg. Salzburg steht hierbei stellvertretend für eine Stadt mit angespanntem Wohnungsmarkt (Zeller et al., 2018). So weist Salzburg 
im österreichweiten Vergleich seit Jahren neben Wien und Innsbruck die höchsten Mieten und Immobilienpreise auf - konkret EUR 10 kalt $^{1}$ (2017) beziehungsweise EUR 4161 (Eigentumswohnung Neubau 2017) je Quadratmeter (WKO, 2002-2018). Die Dynamik ihrer Entwicklung liegt bedeutend über jener der Einkommen, vor allem bei kleineren Wohnungen (Kircher et al., 2018). Gleichzeitig steht Salzburg für eine, auch im europäischen Vergleich, stark touristische Stadt, die erheblich mit den Auswirkungen des Massentourismus ,kämpft“ (Roland Berger, 2018).

Die ökonomische Dimension der Wohnungsfrage zeigt sich ebenfalls in struktureller Hinsicht. Salzburg kann hierbei stellvertretend für Städte mittlerer Größe ohne globale Funktionen gesehen werden, die von jüngeren Prozessen der Finanzialisierung und Responsibilisierung betroffen sind - etwas, das bislang vorwiegend in große Metropolen wie Frankfurt, Hamburg und München beobachtet wurde. Mittlerweile aber sind in zunehmendem Ausmaß auch Mittelstädte, kleinere Universitätsstädte und touristische Regionen interessant für das institutionelle und private anlagesuchende Kapital (Fehlberg and Mießner, 2015; Engel \& Völkers, 2013; VanHametner and Zeller, 2018). Die sich seit mehreren Jahrzehnten vollziehende Bedeutungszunahme finanzieller Verwertungsstrategien und Rentenerträge manifestiert sich auch im Immobiliensektor. Wohnungen als Finanzanlage, zur Vorsorge, wegen niedriger Zinsen oder auch aus Mangel an alternativen risikoarmen Anlagemöglichkeiten sind zunehmend von Bedeutung für Privathaushalte, aber auch für institutionelle Anleger_innen.

Salzburg ist eine stark touristisch geprägte Stadt. Mehr als 3 Millionen Übernachtungen zählt die Stadt mit knapp 156000 Einwohner_innen. Die dafür notwendige touristische Infrastruktur führt zur Verdrängung ${ }^{2}$ von Wohninfrastruktur und -bevölkerung aus dem innerstädtischen Bereich (z.B. Altstadt-Mülln: knapp $15 \%$ Bevölkerungsrückgang von 2008 bis 2017 (Stadt Salzburg, 2013, 2018b; eigene Berechnungen)), Phänomene wie Kurzzeitvermietungen für Tourist_innen aber auch neue Wohnbauprojekte, die explizit für Anleger_innen errichtet werden, verstärken das Problem.

Das Beispiel Salzburg zeigt, wie spezifische wohnungspolitische Handlungsweisen zu einer Verschärfung der Wohnungsfrage führen können. Verständlich werden diese Prozesse nur im Rahmen der institutionellen, politischen und ökonomischen Verhältnisse sowie der konkreten Ausgestaltung der lokalen Wohnpolitik und der mit ihr verbundenen Boden- und Sozialpolitik.

\footnotetext{
${ }^{1}$ Nettomietzins exklusive Betriebs- und Heizkosten für Wohnungen, für welche die Mietzinsobergrenzen des Mietrechtsgesetzes nicht gelten.

${ }^{2} \mathrm{Zu}$ sozialräumlichen Verdrängungsprozessen liegen bislang nur wenige gesicherte Erkenntnisse vor (Bichler, 2018; Schoibl, 2011). Andere Formen der Verdrängung, beispielsweise durch das Auftreten von Gewerbeimmobilien oder Ähnlichem sind weder politisch noch medial ein Thema.
}

Aufgrund der allgemeinen Bedeutungszunahme finanzieller Verwertungsstrategien, der Eigenheiten Salzburgs als touristische Destination und der starken, überdurchschnittlichen Preissteigerungen von Wohneigentum und Mieten drängen sich uns folgende zwei Untersuchungsfragen auf.

- Wie äußert sich die Wohnungsfrage in Salzburg unter den spezifischen ökonomischen und institutionellen Bedingungen?

- Welche Rolle spielen die Ausrichtung der Stadt Salzburg auf den Tourismus, die zunehmend stärkere Orientierung von Wohnraum als Finanzanlage und die (lokalen) politisch-institutionellen Rahmenbedingungen für die Entwicklung der Wohnungssituation in Salzburg?

Diese Fragen lassen sich nicht unabhängig voneinander beantworten, da sich Prozesse und Phänomene gegenseitig bedingen. Darum verbinden wir in unserer Analyse die ökonomischen und institutionellen Veränderungen, wobei wir diese jeweils in den größeren internationalen und nationalen Kontext setzen.

In Abschnitt zwei legen wir dar, wie sich der stärkere Einfluss von Finanzialisierungsprozessen auf dem Wohnungsmarkt zeigen kann. Anschließend reißen wir im dritten Abschnitt das Problem der Touristifizierung an und skizzieren dessen Auswirkungen auf Wohnungsmärkte. Der vierte Abschnitt geht der Frage nach, welche Rolle Planung und regionale Politiken, eingefasst durch nationale institutionelle Rahmenbedingungen, auf die Wohnungsfrage nehmen. Dabei fokussieren wir uns explizit auf jene Politikfelder, die den bereits im zweiten und dritten Abschnitt aufgeworfenen Themen entsprechen. Im fünften Abschnitt zeigen wir, wie in Salzburg der zunehmende Tourismus die Verwertung von Eigentum in Form von Zins- und Rentenerträgen begünstigt. Im sechsten Abschnitt argumentieren wir, wie die gemeinsame Betrachtung von Finanzialisierungs- und Tourismusprozessen wesentlich zum Verständnis von Problemen auf regionalen Wohnungsmärkten beiträgt.

\section{Wohnen als Finanzanlage}

Welchen Einfluss hat die Nachfrage nach Wohnungen als Finanzanlage auf die Entwicklung der Wohnkosten? Mehrere Faktoren haben zur Bedeutungszunahme finanzieller Anlagestrategien im Bereich des Wohnens geführt. Dazu zählen das stärkere Auftreten institutioneller Anleger_innen am Immobilienmarkt und die Ausweitung privater Anlagen in Betongold aufgrund verstärkter Unsicherheiten am Kapitalmarkt, niedriger Zinsen und dem Mangel alternativer, stabiler Anlagemöglichkeiten sowie der (gefühlten) individuellen Verantwortung für die eigene Altersvorsorge (Heeg, 2013). Wesentliche Rahmenbedingungen dafür sind die Liberalisierung der Finanzmärkte und Deregulierung der Immobilienmmärkte einhergehend mit dem Rückzug der öffentlichen Hand, die Zunahme von Verschuldung sowie 
die Errichtung kapitalgedeckter Altersversicherungssysteme. Durch neue Finanzmarktinstrumente wurden Immobilienbestände in standardisierte und vergleichbare Waren zur Kapitalanlage. Diese Rahmenbedingungen erleichtern es Finanzakteuren am Immobilienmarkt Kapital zu platzieren. Das führte zu einer deutlichen Ausweitung von Investmentmöglichkeiten. Wie Aalbers (2016) argumentiert, konnte so das finanzdominierte Akkumulationsregime zunehmend seine Auswirkungen auch auf die Wohnungsmärkte entfalten.

Die jüngere geographische Immobilienwirtschaftsforschung zeigt, dass die Immobilienmärkte großer Metropolen zunehmend durch diese Prozesse - vor allem durch international mobiles und liquides Anlagekapital und durch Auswirkungen der Responsibilisierung - geprägt werden (Heeg, 2013; Dörry, 2010; Fernandez and Aalbers, 2016; Aalbers, 2016). Aber auch kleinere Städte, vor allem regionale Universitätsstädte und sonstige sogenannte B-Lagen sind zunehmend betroffen (Reutter, 2012; Engel \& Völkers, 2013; Fehlberg and Mießner, 2015).

Die Bedeutungszunahme finanzieller Anlagestrategien mit dem Ziel kurzfristiger Profitmaximierung und Renditesteigerung abseits realwirtschaftlicher Produktionsprozesse wird auch als Finanzialisierung bezeichnet. Wir verstehen Finanzialisierung als einen Prozess der Akkumulation von Geldkapital (also aus Geld mehr Geld machen; G-G'), das nach profitablen Anlagemöglichkeiten und neuen Verwertungsfeldern sucht (vgl. auch Chesnais, 2016; Belina, 2018; Serfati, 2011). Zahlreiche Finanzialisierungsprozesse beruhen auf einer starken Bedeutungszunahme von Rentenerträgen auf der Grundlage von Eigentumsmonopolen - also Einkommen ohne direkte Gegenleistung (Zeller, 2008). Rentenerträge sind den Zinserträgen ähnlich. Beide entstehen nicht aus einem produktiven Wertschöpfungsprozess, sondern entstammen einer Umverteilung von Mehrwert begründet auf Eigentumstiteln. Investoren erzielen ihre Renditen in Form von Zinserträgen, zu denen auch Dividenden zu zählen sind, die Eigentümer_innen von Immobilien erzielen Renteneinkommen. Bei einer ungünstigen Verteilung der Marktmacht zwischen Angebot und Nachfrage nach Immobilien bzw. Wohnraum können die Eigentümer_innen überdies eine Monopolrente erzielen (Harvey, 1982:353, 2001). Dieser Akkumulationsprozess außerhalb des Produktionsprozesses nimmt im Immobiliensektor eine ganz besondere Bedeutung ein und kann durch verschiedene Prozesse zwischen Kreditnehmer_innen und Kreditgeber_innen, Mieter_innen und Eigentümer_innen erfolgen (Belina, 2018).

Vor allem institutionelle Investor_innen, zunehmend aber auch Privatpersonen, haben Wohnungen als Anlageobjekte zur Vermehrung ihres Kapitals identifiziert (Heeg, 2013; Scharmanski, 2006; Hesse and Preckwinkel, 2009). Beide Akteursgruppen treten in unterschiedlichen Formen auf den Immobilienmärkten auf. Ihnen ist gemeinsam, dass sie Wohnraum in erster Linie erwerben, um ihr Kapital anzulegen und zu vermehren. Ein Beispiel sind Pensions- und Investmentfonds die ganze Wohnportfolios (für eine bestimmte
Zeit) aufkaufen. Ein weiterer Grund für die wachsende Nachfrage nach Wohneigentum liegt, in der zunehmenden Verunsicherung vieler Menschen in Bezug auf ihre Altersvorsorge (ERGO, 2018) - vor allem in konservativen Wohlfahrtsstaaten (vgl. Esping-Andersen, 2013) mit traditionell ausgeprägten sozialen Sicherungssystemen wie Österreich, Deutschland oder auch Frankreich. Je mehr der Eindruck entsteht, die umlagefinanzierte Altersversicherung reiche nicht mehr, um den Lebensunterhalt im Alter zu finanzieren, desto eher sehen sich viele Menschen motiviert, ihre Altersvorsorge über Wohnungseigentum zusätzlich abzusichern (AXA Deutschland, 2016). Heeg (2013) bezeichnet diesen Prozess, mit dem Menschen zunehmend individuell verantwortlich für die Altersvorsorge gemacht werden und sich dadurch dem Diktat der Finanzmarktlogik unterwerfen als Responsibilisierung. Dieser geht zugleich mit einer Verlagerung privater Sparguthaben einher (Jäger, 2006) und führt dazu, dass mehr Kapital auf der Suche nach Anlagemöglichkeiten ist. Hinzu kommt die Verunsicherung vieler privater Anleger_innen bei Investments am Kapitalmarkt. Das verstärkt die Nachfrage nach so genannten Vorsorge- bzw. Anlagewohnungen, also Wohnungen, welche Anleger_innen nicht selbst bewohnen, sondern zum Zwecke der Wertanlage und Vorsorge erwerben und teilweise vermieten.

Unternehmen und zunehmend auch wohlhabendere Privatpersonen entwickeln und errichten Vorsorge- und Anlagewohnungen (Nothegger, 2018) als Renditeobjekte zur Miete oder für den Verkauf. Großen Teilen der Bevölkerung fehlen für den - vielfach gewünschten - Erwerb von Vorsorge- und Anlagewohnungen allerdings die finanziellen Möglichkeiten (AXA Deutschland, 2016). Dies führt zu einer stärkeren Polarisierung zwischen wenigen Mehrfacheigentümer_innen und vielen eigentumslosen Mieter_innen. Die Quote von Eigentümer_innen, die Selbstnutzer_innen sind, sinkt deshalb seit der Finanzkrise neben vielen europäischen Ländern auch in Österreich (von 59,2 \% im Jahr 2008 auf $55 \%$ im Jahr 2017) (Statistik Austria, 2018). Dieses Phänomen hat auch eine generationelle und demographische Komponente (Helbrecht and Geilenkeuser, 2012). Aktuell wird diese Thematik unter dem Terminus multiple property ownership international - vor allem in Ländern mit traditionell hoher Eigentumsquote - diskutiert (vgl. Ronald and Kadi, 2017).

\section{Der Tourismus und die Wohnungsfrage}

Neben der zusätzlichen Nachfrage nach Immobilien getrieben durch Vorsorge und Anlage unterschiedlicher Akteur_innen, stehen regionale Immobilienmärkte gerade in Städten mit ausgeprägtem Tourismus zusätzlich unter Druck. Am Beispiel Salzburg mit seiner Bedeutung als Tourismusund Kulturstadt zeigen wir auf, wie neben klassischen Städteurlauber_innen, die unterschiedliche touristische Infrastrukturen nutzen, zunehmend auch über den klassischen Städtetourismus hinausgehende externe Nachfragen den re- 
gionalen Wohnungsmarkt unter Druck setzen und die Wohnungsfrage verschärfen. Hinzu kommen Kurzzeitvermietungen, Nebenwohnsitze wohlhabender Gruppen und (teilweise daraus resultierender) Leerstand. Gemein haben alle diese Phänomene, dass sie klassische langfristige Wohnformen in der Stadt Salzburg verdrängen.

Der Städtetourismus hat zuletzt durch rasantes Wachstum neue Dimensionen erreicht. Ein Grund ist die Abkehr vieler Menschen vom klassischen, langen Sommerurlaub hin zu kürzeren (City-)Trips (Hennig, 1999). Tourismus ist für viele Kommunen ein wichtiger Wirtschaftsfaktor (Hopfinger, 2017; Freytag and Glatter, 2017). Traditionell wird die Wirkung des Tourismus vor allem durch die ökonomische Brille betrachtet, die Attraktivität einer Stadt an der Anzahl an Übernachtungen und Konsumausgaben von Urlauber_innen gemessen. Touristischer Konsum in Einzelhandel, Gastronomie und im Beherbergungswesen schafft Wertschöpfung und Arbeitsplätze und wird deshalb politisch unterstützt (Freytag and Popp, 2009).

Zunehmend erfährt der Tourismus aufgrund seiner negativen Begleiterscheinungen wie hohem Verkehrssaufkommen, der Belastung städtischer Infrastrukturen und dem Anstieg der regionalen Lebenshaltungskosten auch Kritik (vgl. Freytag and Glatter, 2017). Die aktuellen Diskussionen um overtourism bzw. zunehmenden lokalen Widerstand, der in zahlreichen Städten zu beobachten ist, zeigen sowohl die politische Dimension des Themenkomplexes Tourismus und Wohnungsmarkt als auch dessen sozialräumliche Relevanz (vgl. Novy and Colomb, 2017; Seraphin et al., 2018). Dienstleistungsangebote passen sich an die Bedarfe von Tourist_innen an, Lebenshaltungskosten steigen, Wohnraum wird für touristische Zwecke verwendet und steht in sinkendem Ausmaß der Bevölkerung zur Verfügung. In diesen Orten kann es nun zur bewussten Ablehnung des Tourismus kommen (Blickhan et al., 2014; Novy and Colomb, 2017). Neben dem Tourismus gibt es noch weitere Treiber sozialräumlicher Differenzierung in Städten, die als klassische Tourismusdestination gelten. Dies betrifft neben Arbeitsmigrant_innen und Pendler_innen immer mehr Personen, die diese Städte als zusätzlichen Wohnstandort nutzen wollen, ohne einen Hauptwohnsitz zu begründen (vgl. Diskussion um amenity migration bzw. Ruhestandsmigration: Gosnell and Abrams, 2011; Engfer, 2018).

Eine Variante dieses Phänomens ist der Zuzug vor allem älterer wohlhabender Menschen aus unterschiedlichen Gründen (Lebensstil, Attraktivität und Image des Zuzugsortes, Gesundheitsaspekten, etc.) in Regionen abseits der großen Metropolen, die durch ihre besondere Attraktivität gekennzeichnet sind. Für Salzburg, werden wir weiter unten argumentieren, sind diese über den Tourismus hinausgehenden Nachfrageprozesse - allerdings nicht nur auf ältere Personen beschränkt - von großer Bedeutung. Mit diesem Migrationsprozess geht auch steigender Druck auf die Wohnungsmärkte einher (Ghose, 2004). Denn vielfach werden Immobilien von den Zuziehenden nur als Zweitwohnsitz verwendet (Sted- man et al., 2006). Gemeinsam ist diesem Prozess und dem zuvor genannten Tourismus, dass Menschen von außerhalb einer Region aufgrund bestimmter Vorzüge in eine Gegend kommen, zusätzliche Nachfrage nach Wohnraum artikulieren, ohne diesen dauerhaft zu nutzen und so die Wohnungsmarktsituation für die lokale Bevölkerung verschärfen.

\section{Institutionelle Rahmenbedingungen der Wohnungspolitik in Salzburg}

Tourismusinduzierte Prozesse und Finanzialisierungsprozesse können nur dort ihre volle Wirkung auf den Wohnungsmarkt entfalten, wo ordnungpolitische Rahmenbedingungen dies zulassen oder begünstigen. Deswegen setzen wir uns im folgenden Abschnitt mit den wesentlichen Elementen der österreichischen Wohnungspolitik anhand der zentralen wohnungspolitischen Instrumente Mietrechtsgesetz (MRG), Wohnbauförderung (WBF) und gemeinnützigem Wohnbau sowie ihrer konkreten Ausgestaltung in Salzburg auseinander. Diese Ausführungen ergänzen wir mit einem Blick auf die regionale Ausgestaltung der Bodenpolitik und der aktiven Wohnraumbereitstellung in Salzburg.

Den Regulierungsindizes der OECD (2011; Rent-ControlIndicator und Tenant-Landlord-Indicator) zufolge ist Österreichs Wohnungsmarkt stark reguliert. Der Mieterschutz gestaltet sich über das Mietrechtsgesetz (MRG) und flankierend das Wohnungsgemeinnützigkeitsgesetz (WGG). Beide regulieren in unterschiedlichem Ausmaß die Festlegung der Mietzinshöhe und der Kündigungsfristen, gelten aber nicht für den gesamten Wohnungsbestand. Ob das MRG voll angewendet wird, ist abhängig vom Errichtungszeitpunkt des Gebäudes und dem Vorliegen von Förderungen. Darüber hinaus richten sich die juristischen Möglichkeiten der Mietzinsvereinbarung nach dem Zeitpunkt des Vertragsabschlusses. Durch mehrere Anpassungen seit den 1990er Jahren wurde die Mietrechtsgesetzgebung eingeschränkt (Kunnert and Baumgartner, 2012). Aufgrund der Bedeutung des Errichtungszeitpunkts für die Anwendung des MRG, geht dessen Relevanz als mietenregulierendes Instrument zunehmend zurück. In der Stadt Salzburg ist die Wirkung des MRG nur gering, da die wesentlichen Anwendungsvoraussetzungen - Errichtung vor 1945 sowie Förderung durch WBF - nur einen kleinen Teil des Wohnungsbestands betreffen. Die klassischen mehrgeschossigen Mietshäuser der Gründerzeit und andere Wohngebäude aus den Bauperioden bis 1945 stellen nur knapp $17 \%$ aller Wohnungen. Der Anteil an geförderten Wohnungen, die einer Mietpreisregulierung des WGG unterliegen, ist mit $21 \%$ am Gesamtbestand im Vergleich zu anderen österreichischen Städten gering (Stadt Salzburg, 2018a; Amann and Lugger, 2016).

Die Wohnbauförderung ist seit über 70 Jahren hinweg das zentrale Steuerungsinstrument zur Schaffung sozialen Wohnbaus in Österreich (Amann and Lugger, 2016:55). Seit den 1990er Jahren kam es allerdings zu mehreren Schwächungen 
des Systems. Die Wohnbauförderung ging in den Zuständigkeitsbereich der Länder über und wurde real gekürzt (Streimelweger, 2010). Die bestehende Zweckbindung wurde aufgehoben und Fördermittel können seitdem von den Ländern auch für Budgetsanierung bis hin zu Spekulation ,zweckentfremdet" werden (Kunnert and Baumgartner, 2012; Streimelweger, 2010).

Durch dies, sowie durch eine Stärkung der Subjekt- gegenüber der Objektförderung wurden die Möglichkeiten der WBF soziale Wohninfrastruktur zu errichten in den letzten Jahren geschwächt (Streimelweger, 2010; Kunnert and Baumgartner, 2012). Hinzu kommen je nach Bundesland unterschiedliche Ausrichtungen der WBF. Im Bundesland Salzburg werden aktuell auch die Errichtung und der Kauf von Immobilien mit einmaligen nicht-rückzahlbaren Zuschüssen gefördert. Insgesamt wurden 2017 hierfür $37 \%$ des gesamten Förderbudgets verwendet (Land Salzburg, 2018). 2018 kam es zu einem deutlichen Rückgang auf nur mehr $26 \%$ (Land Salzburg, 2019). Da die Zuschüsse nicht zurückgezahlt werden müssen, schwächt dies die Refinanzierung der Wohnbaufördermittel. Bislang speisten die Forderungsrückzahlungen einen Teil des zukünftigen WBF-Budgets. Die Förderungen für Mietwohnbau wurden zudem auch gewerblichen Bauträgern zugänglich gemacht, bis 2013 war dies den gemeinnützigen Wohnbauträgern vorbehalten. Diese gewerblichen Bauträger können aber nach 25 Jahren ohne rechtliche Einschränkungen über die geförderten Projekte verfügen - freie Miete oder Verkauf sind möglich. Günstiger Wohnraum wird damit nicht nachhaltig gesichert (Amann and Mundt, 2017).

Neben der Mietenregulierung und staatlicher Wohnbauförderung stellen die gemeinnützigen Bauvereinigungen (GBV) die dritte Säule der österreichischen Wohnungspolitik dar. Ihre zentrale Aufgabe ist, Wohnungen zu errichten, die für kleine und mittlere Einkommen erschwinglich sind (Rechnungshof, 2007). Drei Aspekte definieren das Alleinstellungsmerkmal der gemeinnützigen Bauvereinigungen: Vermögensbindung, Kostendeckung und Ertragssteuerbefreiung (Feichtinger and Schinnagl, 2017). In der Stadt Salzburg stellen die gemeinnützigen Bauvereinigungen vergleichsweise geringe 21,4\% des Wohnungsbestandes (siehe Abbildung 1). Die restlichen Wohnungen werden vorwiegend von privaten Personen gehalten. Allerdings sind die GBV trotzdem ein wichtiger wohnungpolitischer Akteur. Konkret stellen sie die treibende Kraft des Wohnungsneubaus in Salzburg. Knapp 50\% aller neuen Wohnungen wurden in den letzten Jahrzehnten von den GBV errichtet (Straßl et al., 2018). Trotz dieser strukturellen Bedeutung ist ihr Einfluss auf die Preisentwicklung beschränkt, da auch sie an die (lokalen) wohnungsmarktpolitischen Rahmenbedingungen gebunden sind (Baulandpreise, Konkurrenz zu privaten Bauträgern, baurechtliche Auflagen). So sind die Mieten im gemeinnützigen Segment in Salzburg im österreichweiten Vergleich am höchsten (Amann et al., 2014).

Die öffentliche Hand spielt in Salzburg insgesamt nur eine untergeordnete Rolle am Wohnungsmarkt (Bund 0,7\%,
Land 0,4\%, Gemeinde 2,8\% der Wohnungen)(Stadt Salzburg, 2018a). Der städtische Wohnungsbestand wurde im Jahr 2005 ausgegliedert und wird seitdem von einer GBV betreut. Die Stadt hat allerdings das Vergaberecht für einen Teil der Wohnungen der GBV und ist gemeinsam mit dem Land Eigentümer der größten GBV.

Auch Bodenpolitik und Raumplanung haben einen großen Einfluss auf die Ausgestaltung der Wohnungsfrage in Salzburg. Die Verfügbarkeit und Leistbarkeit von Grundstücken ist eine wesentliche Voraussetzung für sozialen Wohnbau. Salzburg ist eine grüne Stadt in der rund $58 \%$ des Stadtgebiets als Grünland gewidmet sind ${ }^{3}$ (Stadt Salzburg, 2018a). Dies liegt vor allem an einer raumplanerischen Besonderheit - der Grünlanddeklaration, welche einen großen Teil des Salzburger Grünlands unter restriktiven und dauerhaften Schutz vor Verbauung und Umwidmung stellt (Dopsch and Hoffmann, 2008). Zugleich reduziert dieser Grünlandschutz die Gestaltungsmöglichkeiten der Stadt, insbesondere die Errichtung von Wohnbau, da er die Verfügbarkeit von Bauland stark einschränkt. Die Dominanz privater Eigentümer und diese restriktive Raumplanung sind entscheidende Komponenten, weshalb weite Teile der Stadt nach wie vor eine geringe Baudichte aufweisen (vgl. Spitzer et al., 2017). Preiswerter Wohnungsneubau findet in der Tat meist auf den bereits intensiv genutzten Stadtteilen im Norden Salzburgs statt. Im privilegierten Salzburger Süden wird Wohnungsneubau vor allem in Form exklusiver Wohnkomplexe errichtet. Dass sich diese Dichotomie auch in den Wohnsitzpräferenzen der Salzburger_innen widerspiegelt, konnte Weichhart (1988) schon vor 30 Jahren nachweisen und belegt damit die Langfristigkeit und Pfadabhängigkeit von materialisierter Wohnpolitik.

\section{Tourismus- und Finanzialisierungsinduzierte Phänomene am Salzburger Wohnungsmarkt}

In diesem Abschnitt erörtern wir, wie sich die Finanzialisierungsprozesse und Prozesse des Tourismus konkret auf den Wohnungsmarkt in Salzburg auswirken. Dabei zeigen wir auf, dass der Tourismus und die Finanzialisierung getrennte Phänomene am Salzburger Immobilienmarkt begründen, manche Phänomene aber dezidiert auf beide Entwicklungen zurückgeführt werden können. Tourismus und Finanzialisierung bedingen sich teilweise auch bzw. begünstigen sich wechselseitig. In Abbildung 2 zeigen wir schematisch auf, welche Phänomene durch Tourismus und Finanzialisierung ausgelöst oder verstärkt werden und stellen diese in den folgenden Abschnitten konkret für Salzburg vor.

Vor allem in der zentralen Altstadt ist die gesamte Infrastruktur auf den Tourismus ausgerichtet. Zweitwohnsitze sind ebenfalls weitgehend durch den Tourismus gepräg-

\footnotetext{
${ }^{3}$ Vergleichbare Landeshauptstädte wie Graz und Linz (je ca. $45 \%$ ) sowie auch die Hauptstadt Wien haben einen geringeren Grünlandanteil.
} 


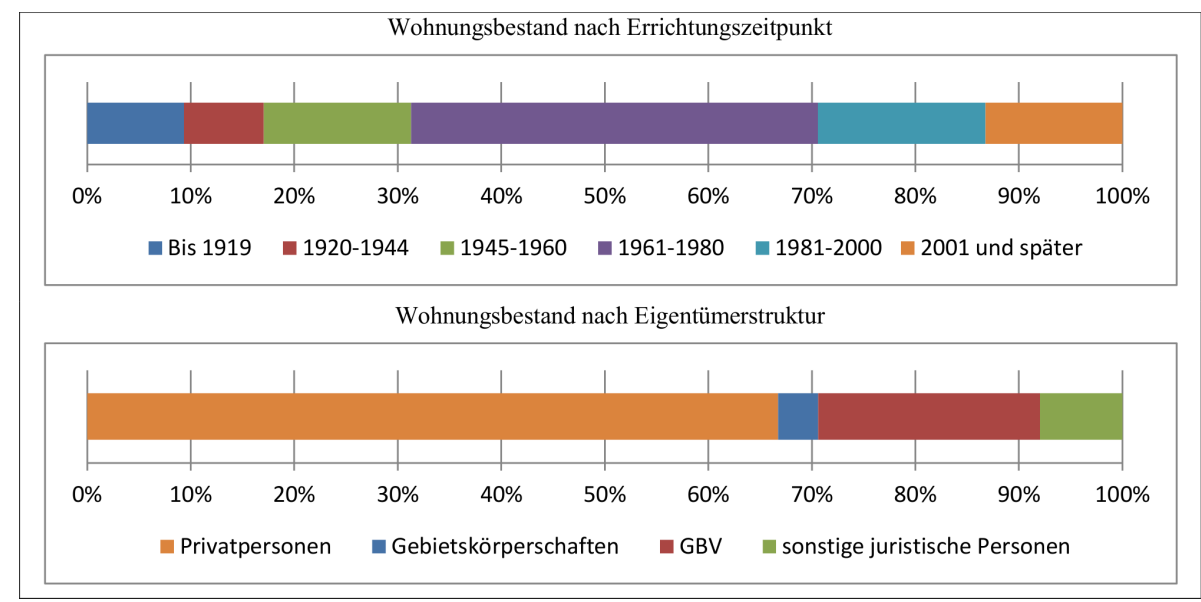

Abb. 1. Struktur des Wohnungsmarkts in Salzburg; Quelle: Stadt Salzburg (2018a).

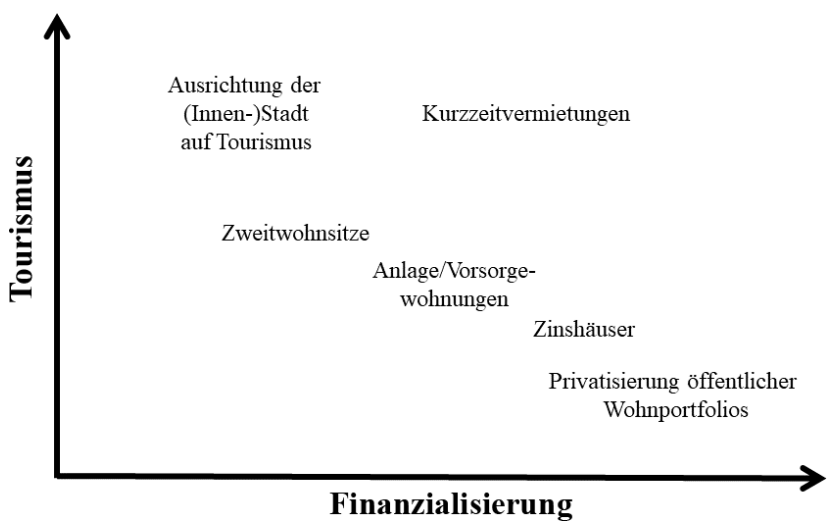

Abb. 2. Tourismus- und finanzialisierungsinduzierte Phänomene am Salzburger Wohnungsmarkt; eigene Darstellung.

te Realität in Salzburg. Fallen Anlageinteressen und touristische Interessen in Salzburg zusammen, sind privat genutzte Vorsorgewohnungen das Resultat. Werden diese nicht selbst genutzt, werden sie entweder vermietet, oder einer häufig lukrativeren (touristischen) Kurzzeitvermietung zugeführt. Größere Kapitalanlagemöglichkeiten, die weniger von touristischen Prozessen betroffen sind, stellen Zinshäuser und die Privatisierung öffentlicher Wohnungsbestände dar. Letzteres allerdings in Salzburg nur in geringem Ausmaß.

\subsection{Manifestation der Finanzialisierung in Salzburg}

So wenig wie die zunehmend marktförmige Organisation des Wohnungsmarktes auf einen singulären Prozess und Begründungszusammenhang zurückgeführt werden kann, so wenig gibt es auch nur ein daraus abgeleitetes Resultat. Wie wir im zweiten Abschnitt gezeigt haben, manifestiert sich „Finanzialisierung " in unterschiedlichem Ausmaß, je nach handelnden Akteuren und Motivlagen. Gemein ist allen nur, dass sie Wohnobjekte nicht (nur) durch die Brille der Bedürfnis- befriedigung betrachten, sondern als Anlageobjekt ansehen. Konkret zeigt sich die Finanzialisierung also zum einen bei der Nachfrage respektive den Nachfragemustern nach Immobilien durch Privatpersonen wie auch Unternehmen. Zum anderen ist aber auch ein darauf differenziertes Angebot sowie eine andere Akteursstruktur bei Projektentwicklung, Bau und Vertrieb offenkundig (vgl. Romainville, 2017).

Im Gegensatz zu zahlreichen deutschen Städten, wurden in Salzburg kaum öffentliche Wohnungsbestände an institutionelle Investor_innen veräußert. Dies liegt in Salzburg daran, dass hier im Gegensatz beispielsweise zu Wien kein nennenswerter kommunaler Wohnbau besteht, der hätte privatisiert werden können. Mittelgroße Portfolios in privater Hand bestehen dennoch. Einerseits führte die Privatisierung der bundeseigenen Wohnbauträger BUWOG und BIG auch in Salzburg zur Veräußerung einiger hundert Wohnungen. Andererseits realisierten in der Vergangenheit private Immobilienentwickler in Salzburg Bauprojekte selbst, zwar vorwiegend für den Verkauf, teilweise aber vermieteten die Bauträger die errichteten Wohnungen auch.

Der Boom großer Mietwohnhäuser als Renditeobjekte (in Österreich auch als Zinshäuser bezeichnet) bringt ebenfalls die Nutzung von Wohnraum als finanzielle Anlageform zum Ausdruck. Diese Renditeobjekte bieten die Möglichkeit Kapital in bereits errichteten Wohnraum anzulegen und über die Mieteinnahmen Rentenerträge zu erwirtschaften. Der österreichische Markt hatte 2017 ein jährliches Transaktionsvolumen von knapp EUR 1,75 Milliarden und weist eine deutliche Dynamik auf - von 2016 auf 2017 stiegen die Transaktionen in Österreich um 17,5\%. Salzburg verzeichnet trotz seiner geringen Größe im Verhältnis zu den anderen Bundesländern ein überproportional großes Transaktionsvolumen mit vielen kleineren Transaktionen (Hudej Zinshäuser, 2018). Interessant ist, dass sich durch die Transaktionen nicht nur individuelle Eigentumsrechte ändern, sondern insgesamt eine Änderung der Eigentumsstrukturen vollzieht. In Salzburg, wie auch in vielen anderen Bundesländern sind der überwie- 
gende Teil der Verkäufer_innen Privatpersonen, hingegen die Käufer_innen überwiegend Firmen. Dies unterstreicht das Interesse des institutionellen Anlagekapitals für Immobilien (Hudej Zinshäuser, 2018).

Aber nicht nur ganze Häuser, sondern auch einzelne Wohnungen werden $\mathrm{zu}$ Vorsorge- beziehungsweise Anlagezwecken erworben. Denn die Skepsis bzgl. der staatlichen Pensionsvorsorge ist auch in Salzburg groß. $75 \%$ der Salzburger erwarten, ihren Lebensstandard mit staatlicher Pension alleine nicht halten zu können (IMAS, 2019). Die genaue Abgrenzung zwischen dem klassischen Wohnungserwerb zur Eigenversorgung und dem Erwerb zu Vorsorgebeziehungsweise Anlagezwecken ist in methodischer Hinsicht jedoch schwierig, da nur über die Motivation des Käufers/der Käuferin erkennbar. Anlagewohnungen werden entweder mit ausschließlicher Vermietungsabsicht, mit Vermietungsabsicht und anschließender Selbstnutzungsperspektive (z.B. in der Pension) oder ausschließlich als Anlage mit der Spekulation auf einen gesteigerten Wohnungswert gekauft. Besonders geeignet dafür sind Wohnungen, die hohe dauerhafte Vermietungschancen haben und damit kontinuierliche Mieteinnahmen garantieren. Deshalb handelt es sich häufig um kleinere (2-3 Zimmer) Wohnungen in Ballungszentren in zentralen Stadtteilen, die entweder Neubauten oder generalsaniert sind.

Zahlreiche Indizien deuten darauf hin, dass es in Österreich zu einem Boom von Angebot und Nachfrage nach Anlage-/Vorsorgewohnungen kommt. Regional differenzierte Daten liegen aktuell nur für Wien vor und zeigen einen deutlichen Nachfrageanstieg. Marktstudien (EHL, 2018), welche auf die besondere steuerliche Behandlung von Wohnungen zur Anlage als Analysekriterium referenzieren, gehen für 2017 von 950 Wohnungen aus, die für Vorsorgezwecke bzw. Anlagezwecke angekauft wurden (ein deutlicher Anstieg von 515 im Jahr 2015). Für 2018 wird ein Anstieg prognostiziert. Diese 950 entsprechen ca. $7 \%$ aller Eigentumswohnungskäufe in Wien 2017.

Generell gibt es in Österreich eine starke Zunahme des Kaufs von Eigentumswohnungen. Dies ist auch in Salzburg - mit zuletzt rückläufiger Tendenz - der Fall (siehe Abb. 3). Dies zeigen wir detailliert in Abschnitt 5.3. und belegen wir anhand einer Analyse aktueller Marktberichte der lokalen und nationalen Immobilienbranche (Hölzl \& Hubner, 2018; WKO, 2002-2018).

Vor allem das Segment der kleinen Wohnungen, die für Anleger besonders interessant sind, boomt. Die stark steigenden Preise im Segment der kleinen Ein- und Zwei-RaumWohnungen können auch als eine verstärkte Nachfrage aus finanziellen Anlagemotiven interpretiert werden. Diese Annahme wird durch Aussagen von Marktakteuren bestätigt (OVI, 2017). Ansonsten kann die Motivation zur Anlage und Vorsorge beim Immobilienkauf nur durch Befragungen belegt werden: $47 \%$ aller Wohnungseigentümer sehen ihr Ei- gentum als Wertanlage bzw. Vorsorge (Sparkasse Salzburg, $2018)^{4}$.

Wie schon oben zu Österreich dargelegt, führen diese Prozesse zu einem Rückgang der Eigentumsquote, eine kleinere Anzahl an Personen wohnt in Ihren ,eigenen“ vier Wänden. Auch im Bundesland Salzburg ist die Eigentumsquote von 2008 auf 2017 von 54,5\% auf 51,7\% gesunken (Statistik Austria, 2018). Die starke Nachfrage nach Anleger- und Vorsorgewohnungen führt dazu, dass dieses Segment auch im Neubau eine immer größere Rolle einnimmt. Bereits 2015 waren $10 \%$ aller neuen Wohnprojekte in Wien reine Vorsorgeprojekte und weitere $19 \%$ solche mit einer Mischung aus Selbstnutzung und Vorsorge (Putschlögl, 2015) und auch in Salzburg werden zunehmend mehr Eigentumswohnungen explizit zur Vorsorge und Anlage erreichtet und beworben. Gerade aufgrund dieser starken Nachfrage und den damit einhergehenden steigenden Preisen kommt es allerdings auch zu einem Rückgang der Renditen, in Salzburg liegen diese nur mehr bei 1,5\% bis $2 \%$ (Jezek, 2019).

\subsection{Tourismus und der Wohnungsmarkt in Salzburg}

Salzburg ist Inbegriff einer klassischen Kulturtourismusdestination und zählt mit seiner 200jährigen touristischen Geschichte zu den ältesten Tourismusregionen. Bereits in den 1920er Jahren des letzten Jahrhunderts gehörte deren Besuch ,zum Kanon des europäischen Bildungsbürgertums und zu den Vergnügungsritualen des Industrieadels“ (Hoffmann and Luger, 1997:33). Gemeinsam mit den Festspielen, bilden die barocke Altstadt, Mozart, ,The Sound of Music" und der Christkindlmarkt ein Amalgam, das sehr viele Tourist_innen anzieht. 2017 verzeichnete die Stadt Salzburg bei knapp 1,75 Millionen Tourist_innen erstmals über drei Millionen Übernachtungen (Stadt Salzburg, 2019) sowie geschätzte neun Millionen Tagestourist_innen (Neuhold, 2018a). Dies bedeutet eine deutliche Steigerung der Nächtigungen von $8 \%$ im Vergleich zum Vorjahr sowie sogar um $81 \%$ seit 2001 (siehe auch Abbildung 4) (Stadt Salzburg, 2019). Salzburg liegt mit 20 Übernachtungen je Einwohner_in sowohl national als auch im internationalen Vergleich ähnlich großer touristischer Destinationen Europas (mit über 1 Million Übernachtungen) auf Rang eins in punkto Touristen_innendichte (Roland Berger, 2015). Die touristische Infrastruktur und Nachfrage sind in Salzburg stark auf das historische Zentrum konzentriert. Stadtstrukturelle Transformationsprozesse sind vor allem im touristischen Stadtkern wahrzunehmen. Die Attraktivität ist für Salzburg nicht nur Segen, sondern auch Fluch. Die Masse an Tourist_innen und vor allem die immer kürzere Verweildauer von durchschnitt-

\footnotetext{
${ }^{4}$ Die Notariatskammer (2016) ließ in einer österreichweiten Untersuchung wesentliche Motive zum Eigentumserwerb abfragen: Neben der Schaffung eines Eigenheims (71\%) spielen Geldanlage (41\%), Altersvorsorge (32\%), potentielle Mieteinnahmen (24\%) und die Vorsorge für Nachkommen (22\%) wesentliche Begründungen beim Immobilienerwerb.
} 


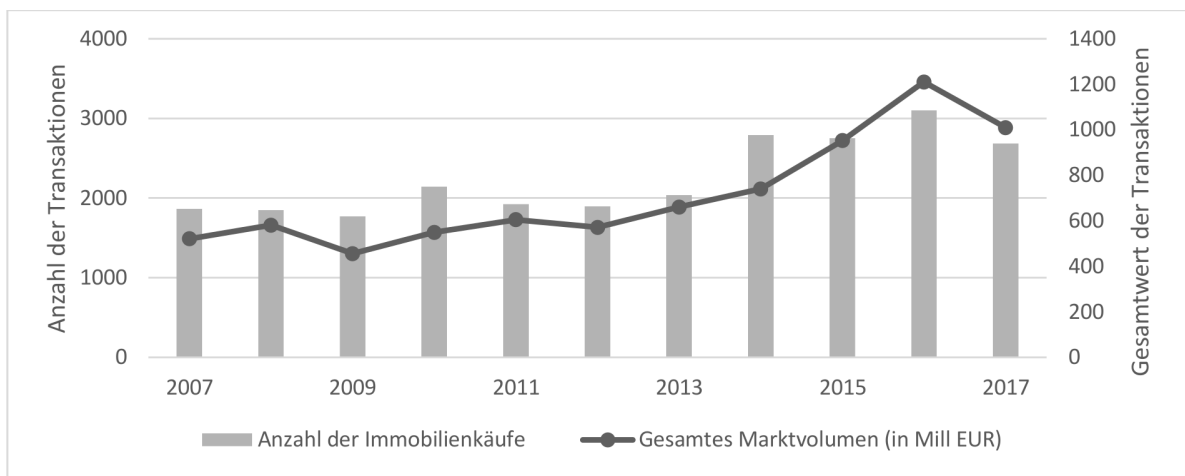

Abb. 3. Steigende Nachfrage nach Eigentum am Salzburger Immobilienmarkt; Quelle: Hölzl and Hubner (2018).

lich unter zwei Tagen, führt dazu, dass die Belastungsgrenzen Salzburgs erreicht werden und sich erste Formen zivilgesellschaftlichen Protests bilden (Hoffmann and Luger, 1997; Stadt Salzburg, 2019; Neuhold, 2018b).

In Salzburgs Zentrum führt die Tourismuskonzentration zu einer Transformation des urbanen Lebensraums, zu Verkehrsproblemen und einer verminderten Lebensqualität. Auch das Einzelhandels- und Dienstleistungsangebot passt sich an. Auf der einen Seite stellen Tourist_innen mittlerweile ein Drittel der Konsumnachfrage in der Innenstadt, zum anderen macht gerade die hohe Touristendichte das Zentrum für Einheimische unattraktiv (CIMA, 2015, 2016). Dies führt zu einer kontinuierlichen Abwanderung der Wohnbevölkerung im touristischen Zentrum (vgl. Hoffmann and Luger, 2011). In der zentralen Altstadt ging die Wohnbevölkerung (Hauptwohnsitz) in den letzten 10 Jahren um knapp $15 \%$ zurück (Stadt Salzburg, 2013; 2018b; eigene Berechnungen), während die Einwohnerzahl der gesamten Stadt im gleichen Zeitraum um knapp $3 \%$ anstieg.

Wohnraum wird im zunehmenden Maße durch andere Nutzungsformen genutzt, die nicht durch einen dauerhaften Wohnsitz begründet sind - im Stadtzentrum stark, in anderen Stadtteilen in abgeschwächter Form. Dabei handelt es sich erstens um touristische Nutzungen, zweitens um darüber hinaus gehende Formen von Zweitwohnsitzen und drittens um leerstehende Wohnungen. Die zusätzliche Nachfrage nach Wohnungen steht in unmittelbarer Konkurrenz zur einheimischen Bevölkerung.

In allen drei Fällen werden diese Wohnungen nicht als Hauptwohnsitz genutzt - dies bedeutet, dass in diesen Wohnungen niemand seinen Lebensmittelpunkt hat. Bereits im Jahr 2011 gab es in Salzburg 14791 Wohnungen ohne Hauptwohnsitzmeldung, das entsprach 17,1\% aller Wohnungen und mehr als einer Verdopplung seit 1991 (Straß1 et al., 2018). Im Vergleich zu anderen österreichischen Landeshauptstädten liegt hier Salzburg, neben Innsbruck mit 18,3\% vor Graz $(15,8 \%)$ und Linz $(13,7 \%)$. Vor allem die Dynamik der Jahre von 2001 bis 2011 mit einer Zunahme um über 5000 Wohnungen ohne Hauptwohnsitz zeigt die Rele- vanz für den lokalen Wohnungsmarkt auf. Seit dem letzten Zensus 2011 gibt es hierzu allerdings keine genaueren Daten. Andere Datengrundlagen, wie etwa der dem Melderegister entnommene Anstieg der Personen mit gemeldetem Nebenwohnsitz in Salzburg, lassen eine Kontinuität der Dynamik vermuten $^{5}$. Alleine zwischen 2003 und 2018 stieg die Anzahl der Einwohner_innen mit Nebenwohnsitz um $26 \%$ (vgl. Tabelle 1) (Stadt Salzburg, 2018a).

Immer öfter werden Wohnungen für touristische Kurzzeitvermietungen über Airbnb und andere Portale zweckentfremdet. Eine aktuelle Studie zählt 700 aktive Angebote alleine auf dieser Plattform, die sich zudem stark auf die innerstädtischen Stadtviertel konzentrieren. $75 \%$ sind ganze Wohnungen oder Häuser, von denen zudem mehr als $50 \%$ dauerhaft dem Wohnungsmarkt entzogen sind (Hof et al., unveröffentlichter Projektbericht). Dies übertrifft bspw. die Zahlen für Wien, wo mittels gleicher Methodik eruiert wurde, dass „nur“ $38 \%$ der über Airbnb angebotenden Wohnungen und Häuser dauerhaft dem Wohnungsmarkt entzogen sind (Seidl et al., 2017). Des Weiteren dominieren kommerzielle Anbieter_innen den Airbnb-Markt in der Stadt Salzburg, die mehrere Angebote haben. Konkret gehen $90 \%$ der Einnahmen an Anbieter_innen, die monatlich mehr als EUR 1500 mit Airb$\mathrm{nb}$ verdienen. Vor allem Top-Anbieter_innen mit mehr als fünf Angeboten zeugen von dieser kommerziellen Struktur. Sie repräsentieren zwar nur $5 \%$ der Airbnb-Anbieter in Salzburg; stehen aber für $28 \%$ der Angebote (Hof et al., unveröffentlichter Projektbericht). Außerdem äußerten sowohl Homesharer als auch kommerzielle Anbieter_innen im qualitativen Teil der genannten Studie übereinstimmend, dass Mehreinnahmen der Hauptgrund der Kurzzeitvermietung sind.

Dieses Angebot steht in direkter Konkurrenz zu einheimischen Wohnungssuchenden, da es sich mehrheitlich um nicht speziell für touristische Zwecke gewidmete Wohnungen, sondern regulären zweckentfremdeten Wohnraum han-

\footnotetext{
${ }^{5}$ Wichtig ist die Unterscheidung zwischen Einwohnern mit „Nebenwohnsitz“, welche auf der möglichen Angabe ,weiterer Wohnsitz" auf den Meldezetteln basiert und Wohnungen ohne Hauptwohnsitzangabe (Nebenwohnsitzwohnungen) (vgl. König, 2017)
} 


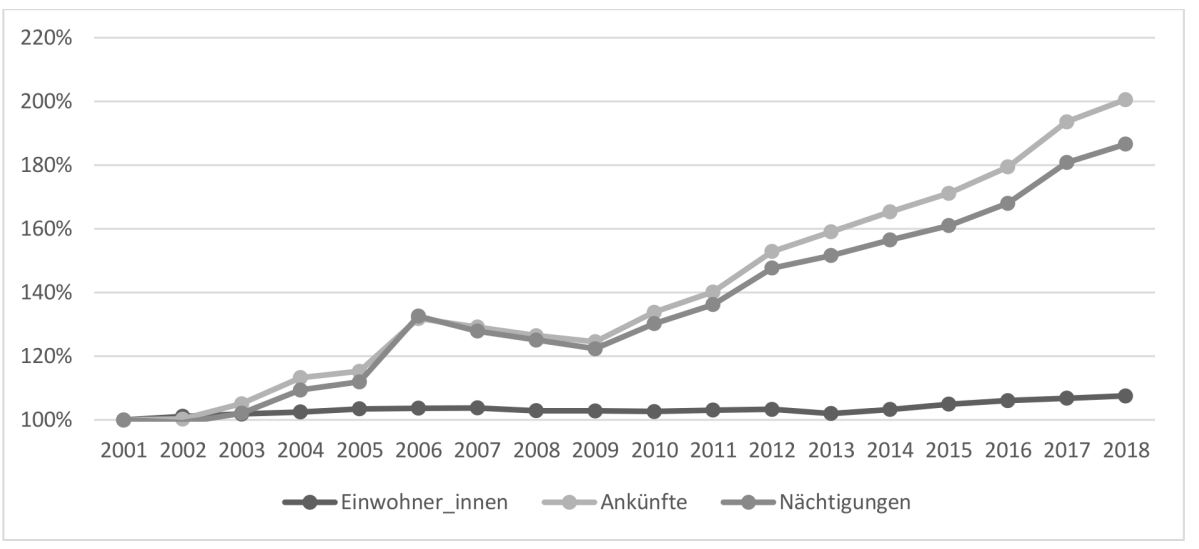

Abb. 4. Starke Zunahme von Ankünften und Nächtigungen in der Stadt Salzburg; Quelle: Stadt Salzburg (2019).

Tabelle 1. Wohnungen und Einwohner_innen mit Haupt-/Nebenwohnsitz; Quelle: Stadt Salzburg (2018a).

\begin{tabular}{lcrrl}
\hline $\begin{array}{l}\text { Index }=1991 ; 2002 \text { massive Bereinigung des } \\
\text { Melderegisters; }{ }^{*} \text { Entwicklung mit Index 2003 }\end{array}$ & 1991 & 2001 & 2011 & 2018 \\
\hline Wohnungen mit Hauptwohnsitz & 100 & 108 & 117 & - \\
Wohnungen ohne Hauptwohnsitz & 100 & 134 & 208 & - \\
\hline Einwohner_innen mit Hauptwohnsitz & 100 & 101 & 105 & 110 \\
Einwohner_innen mit Nebenwohnsitz & 100 & 107 & $71\left(121^{*}\right)$ & $75\left(126^{*}\right)$ \\
\hline
\end{tabular}

delt und Wohnungen ohne, aber auch mit Hauptwohnsitz betrifft. 55\% der Anbieter_innen bieten zudem mehrere Unterkünfte an. Die genannte Studie zeigt, dass die Mehrzahl hierbei professionelle Beherbergungsunternehmer_innen sind. In der Statistik erscheinen diese Wohnungen dann zumeist ohne Hauptwohnsitz.

Immer mehr Menschen leben darüber hinaus nur zeitweilig in Salzburg, weil sie hier studieren und arbeiten oder aber auch, weil Salzburg aufgrund seiner Attraktivität für sie einen weiteren Wohnsitz wert ist (vgl. Weichhart, 1988). Anfang 2019 hatten 21.344 Einwohner_innen der Stadt Salzburg nur einen Nebenwohnsitz in der Stadt Salzburg. Die Gesamtzahl der Nebenwohnsitze stieg seit 2003 kontinuierlich, gleichzeitig änderte sich deren strukturelle Zusammensetzung. Während die Anzahl der österreichischen Staatsangehörigen mit Nebenwohnsitz von 2007 auf 2019 von 14.871 auf 13.593 leicht abnahm, stieg die Anzahl der Nebenwohnsitze anderer Staatsangehörigkeiten deutlich: von $2838 \mathrm{im}$ Jahr 2007 auf 7751 im Jahr 2019. Mit Blick auf die Demographie, lässt sich außerdem eine stark überdurchschnittliche Entwicklung bei den Altersklassen zwischen 50-59 und 70-79 Jahren feststellen. In diesen Altersklassen verdoppelte sich die Anzahl der Nebenwohnsitze von 2007-2017, auch bei den 60-69-Jährigen gab es eine Steigerung um $39 \%$ während die Gesamtzahl im gleichen Zeitraum nur um $21 \%$ stieg (vgl. Tabelle 2). Im Gegensatz dazu entwickelten sich die Hauptwohnsitze in allen Altersgruppen deutlich schwä- cher, auch in den höheren Altersgruppen (Stadt Salzburg, 2018a).

Dies ist ein deutliches Indiz, dass es sich bei der Entwicklung der Nebenwohnsitze nicht nur um ein Phänomen der Ausbildungs- und Arbeitsmigration handelt. Die Nebenwohnsitzfälle verteilen sich nicht nur ungleich auf unterschiedliche Altersgruppen, sondern sind auch ungleichmäBig über die Stadt verteilt. In der zentralen Altstadt sind Nebenwohnsitze doppelt so häufig, wie in der restlichen Stadt. Ähnlich verhält es sich auch im direkt angrenzenden Bereich (Mönchsberg/Inneres Nonntal/Leopoldskron). Spitzenreiter ist das gründerzeitlich geprägte Andräviertel, in dem auf 10 Hauptwohnsitze fast 4 Nebenwohnsitze entfallen. Unterdurchschnittliche Nebenwohnsitzdichten finden sich dafür im ländlichen Zählbezirk Hellbrunn sowie den großen Wohnbezirken außerhalb der touristischen Hotspots wie Maxglan, Liefering, Sam oder Taxham. Dies ist allerdings kein neues Phänomen. Schon in den frühen 1990er Jahren wurden Bedenken laut, die Altstadt könne zur ,Sommeroder Kulturresidenz" für wohlhabende Ausländer verkommen (vgl. Schorn, 1992). Gerade bei den genannten Stadtvierteln handelt es sich um jene mit den höchsten Mieten sowie Eigentumspreisen, was ebenfalls gegen die Evidenz von Studierenden und Pendler_innen spricht. Naheliegender ist deshalb, wie auch oftmals medial kolportiert (vgl. Die Presse, 2016), dass es sich um Wohnsitze wohlhabender Ausländer_innen, vorwiegend aus EU-Ländern aber auch um Österreicher_innen aus anderen Bundesländern handelt, die auf- 
Tabelle 2. Entwicklung der Neben- und Hauptwohnsitze nach Altersgruppen, Entwicklung 2007-2017; Index 2007=100/Quelle: Stadt Salzburg (2018a).

\begin{tabular}{|c|c|c|c|c|c|c|c|c|c|c|}
\hline Altersgruppe & $\sum$ & $0-9$ & $10-19$ & $20-29$ & $30-39$ & $40-49$ & $50-59$ & $60-69$ & $70-79$ & $80<$ \\
\hline \multicolumn{11}{|c|}{ Nebenwohnsitze } \\
\hline absolut & +3796 & -70 & -113 & +221 & +652 & +620 & +1432 & +444 & +546 & +64 \\
\hline $\begin{array}{l}\text { Hauptwohnsitz } \\
\text { relativ }\end{array}$ & 102 & 99 & 101 & 109 & 94 & 90 & 108 & 96 & 146 & 102 \\
\hline absolut & +3497 & -172 & +74 & +1714 & -1323 & -2476 & +1657 & -765 & +4657 & +131 \\
\hline
\end{tabular}

grund der Vorzüge der Stadt einen Zweitwohnsitz begründeten. In Salzburg sind allerdings nur knapp 600 Zweitwohnsitze gemeldet (Straß1 and Riedler, 2015).

Neben der kurzfristigen touristischen Nutzung sowie der Verwendung als Nebenwohnsitz spielt auch unterschiedlich motivierter Leerstand von Wohnungen eine Rolle zur Erklärung der vielen Wohnungen ohne Hauptwohnsitz. Straß1 und Riedler (2015) kommen zum Schluss, dass neben den zeitlichen Mindernutzungen wie kurzzeitvermieteter Wohnungen und räumlichen Mindernutzungen (z.B. ältere Personen in zu großen Wohnungen) ein Bestand von ungefähr 3500 Wohnungen mobilisierbarer Leerstand ist. Sie werden nicht genutzt, weil die Eigentümer_innen eine Vermietung scheuen oder aus finanziellen Gründen nicht für notwendig erachten.

\subsection{Teures Pflaster Salzburg}

Unterschiedliche Formen der touristischen Nutzung von Wohraum, die wachsende Anzahl von Zweitwohnungen, und die Nachfrage nach Immobilien zur Anlage setzen den Wohnungsmarkt in Salzburg unter Druck. Neben anderen Faktoren ließen sie die Nachfrage nach Immobilien bzw. Wohnraum in Salzburg ansteigen. Im Jahr 2016 kletterte die Anzahl an Immobilienverkäufen im Großraum Salzburg auf das Allzeithoch von 3102. Das war ein Plus von 12,6\% gegenüber dem Vorjahr und ein Anstieg um $75 \%$ seit 2009. Auch das Umsatzvolumen der Immobilien stieg von 456 Millionen im Jahr 2009 auf 1,21 Milliarden im Jahr 2016. Wohnimmobilien (Neubauwohnungen, Bestandswohnungen und Häuser) nahmen hier mit 62,5\% einen großen Teil ein. 2017 erfolgte dann ein leichter Rückgang (Hölzl \& Hubner, 2018).

Die jahrelange Zunahme der Nachfrage nach Wohnraum trägt zur Steigerung der Immobilienpreise bei (siehe Tabelle 3). Die etablierten Eigentümer_innen können dies einerseits als Wertsteigerung verbuchen und andererseits nun durch steigende Mieten höhere Rentenerträge erzielen. Die Preise für Neubauwohnungen stiegen von 2007 bis 2017 von durchschnittlich EUR 3478 pro $\mathrm{m}^{2}$ um knapp $20 \%$ auf EUR 4161 pro $\mathrm{m}^{2}$. Bestandswohnungen verteuerten sich im selben Zeitraum sogar um 40\% (WKO, 2002-2018). Die Preise unterscheiden sich je nach ihrem Mikrostandort am Stadtgebiet. Vor allem jene Stadtteile weisen besonders ho- he Immobilienpreise auf, die auch stärker touristisch geprägt sind (Hölzl \& Hubner, 2018). Das heißt die touristischen Verwertungsmöglichkeiten in Verbindung mit der relativen Verknappung des Wohnraums tragen dazu bei, dass die Verwertung von Immobilieneigentum bedeutender wird.

Die Mieten stiegen von 2007 auf 2017 nur um knapp 15\% von EUR 8,7 auf EUR 10 Kaltmiete. Der niedrigere Anstieg der Mieten im Gegensatz zu den Eigentumsimmobilien ist darauf zurückzuführen, dass für Mieten stärkere regulatorische Vorschriften bestehen (siehe Abschnitt 4) und Tourismus und Finanzanlagen stärker den Eigentumsimmobilienmarkt betreffen. Vereinzelt können auch Mieter_innen über Angebote an Vermittlungsplattformen wie Airbnb von touristischer Nachfrage profitieren. Vornehmlich ist Airbnb aber nicht Plattform für Privatanbieter, sondern in bedeutendem Maße Vertriebskanal kommerzieller Anbieter von Ferienoder Geschäftsunterkünften (Hof et al., unveröffentlichter Projektbericht; Kadi et al., 2018). Das schnelle Wachstum von Airbnb-Angeboten bringt die Bedeutungszunahme finanzieller Verwertungsstrategien besonders zum Ausdruck. Mit ihren Investitionen in Immobilien beabsichtigen sie Gewinne aus der Vermietung von Wohnungen zu erzielen.

Von den Preissteigerungen ebenso betroffen ist der Markt für Baugrundstücke. Sowohl unterschiedliche Bauträgerformen als auch Privatpersonen fragen Grundstücke für Wohnbau stark nach. Das Grundstücksangebot ist allerdings begrenzt. Die durchschnittlichen Quadratmeterpreise für Bauland im Großraum Salzburg haben sich von 2007 (EUR 482) bis 2017 (EUR 871) deutlich gesteigert. Das Preisniveau im Hochpreissegment (3. Quartil) liegt 2017 mit durchschnittlich EUR 974 nochmals darüber. Gerade im Vergleich mit anderen österreichischen Städten wie Linz (EUR 319) oder Graz (EUR 237) weist Salzburg mit Innsbruck (EUR 799) eine relative Alleinstellung auf (WKO, 2002-2018; Hölzl \& Hubner, 2018).

\section{Fazit}

Zwei zentrale Entwicklungen haben die Nachfrage nach Wohnraum in den letzten Jahren global gesteigert: Im Zuge der durch zahlreiche institutionelle Veränderungen bewirkten Bedeutungszunahme des konzentrierten Anlagekapitals er- 
Tabelle 3. Teures Pflaster - Kennzahlen des Wohnungsmarktes Salzburg; (WKO, 2002-2018; ÖVI, 2018)

\begin{tabular}{|c|c|c|c|c|c|c|c|c|c|c|}
\hline & \multicolumn{2}{|c|}{ Graz } & \multicolumn{2}{|c|}{ Innsbruck } & \multicolumn{2}{|c|}{$\operatorname{Lin} z$} & \multicolumn{2}{|c|}{ Salzburg } & \multicolumn{2}{|c|}{ Wien* } \\
\hline & 2007 & 2017 & 2007 & 2017 & 2007 & 2017 & 2007 & 2017 & 2007 & 2017 \\
\hline $\begin{array}{l}\text { Immobilienmarkt } \\
\text { (Anzahl der Käufe von } \\
\text { Wohnimmobilien) }\end{array}$ & $\begin{array}{r}3017 \\
(2010)\end{array}$ & 3307 & $\begin{array}{r}1538 \\
(2010)\end{array}$ & 1204 & $\begin{array}{r}1115 \\
(2010)\end{array}$ & 1341 & $\begin{array}{r}1405 \\
(2010)\end{array}$ & 1175 & - & - \\
\hline $\begin{array}{l}\text { Kaltmiete } \\
\text { (in Euro pro Quadratmeter) }\end{array}$ & 6,8 & 8,2 & 8,5 & 11,3 & 7,1 & 8,3 & 8,7 & 10 & - & 9,8 \\
\hline $\begin{array}{l}\text { Neubauwohnungen } \\
\text { (in Euro pro Quadratmeter) }\end{array}$ & 2405 & 2922 & 2680 & 3800 & 2312 & 2735 & 3478 & 4161 & - & 3886 \\
\hline $\begin{array}{l}\text { Bestandswohnungen } \\
\text { (in Euro pro Quadratmeter) }\end{array}$ & 1420 & 1708 & 1924 & 2841 & 1469 & 1710 & 1945 & 2728 & - & 2723 \\
\hline $\begin{array}{l}\text { Baugrundstücke } \\
\text { (in Euro pro Quadratmeter) }\end{array}$ & 193 & 237 & 490 & 799 & 252 & 319 & 482 & 871 & - & 600 \\
\hline
\end{tabular}

* Die Transaktions- und Preisdaten für 2007 liegen den Autoren nur auf Bezirksebene vor, da Wien aus mehreren Bezirken besteht, stehen keine Angaben für die Gesamtstadt zur Verfügung.

langten Immobilien vor allem in den Metropolregionen verstärkt die Rolle einer Kapitalsenke. Zuletzt gerieten zunehmend auch kleine und mittelgroße Städte ins Interessenfeld des anlagesuchenden Kapitals. Hinzu kommt, dass auch Privatpersonen aufgrund verstärkter Unsicherheiten am Kapitalmarkt, niedriger Zinsen und dem Mangel alternativer, stabiler Anlagemöglichkeiten sowie der (gefühlten) individuellen Verantwortung für die eigene Altersvorsorge verstärkt Wohneigentum zur Anlage und Vorsorge erwerben. Diese Tendenz zur Anlage in Wohnraum manifestiert sich regional differenziert.

In ausgeprägten Touristenstädten wie Salzburg kommt mit der starken Zunahme des Städtetourismus noch eine zweite Entwicklung hinzu, welche den Wohnungsmarkt unter Druck setzt. Salzburg ist Beispiel für eine Stadt in der overtourism und seine negativen Auswirkungen für die Einwohner Realität sind. Der Tourismus in Salzburg fördert Prozesse, die in ihrer Summe Wohnraum zweckentfremden und reduzieren. Beiden Entwicklungen immanent ist es Wohnraum ausschließlich als Ware zu sehen und damit die Diskrepanz zum Wohnen als Grundbedürfnis zu verschärfen.

Tourismusinduzierte Prozesse und Finanzialisierungsprozesse können nur dort ihre volle Wirkung auf den Wohnungsmarkt entfalten, wo ordnungpolitische Rahmenbedingungen dies zulassen oder begünstigen. Ihre Wirksamkeit wird durch lokale Prozesse mitbeeinflusst. Die zentralen ordnungspolitischen Instrumente der österreichischen Wohnungspolitik sind in Salzburg verhältnismäßig schwach. Die Instrumente des Mieterschutzes entfalten in Salzburg nur bedingt ihre Wirkung. Dies lässt Investitionen in den Wohnungsmarkt attraktiver werden. Die Veränderungen der Wohnbauförderung verhindern, dass diese einen starken Kontrapunkt zur Marktdynamik darstellt. Der geringe Anteil von gemeinnützigem und öffentlichem Wohnbau in Salzburg gibt den privaten Kapitalinteressen viel Raum.

In Salzburg kommt hinzu, dass die Wohnungsfrage mit Wohnungsnot und hohen Preisen seit jeher besteht. Die Politik hat dieses Problem nie ernsthaft und persistent angegangen (Weichhart, 1988). Die Stadtpolitik fordert und fördert den quantitativen Tourismus und widersetzt sich der Nachfrage nach Wohnraum zu Anlagezwecken nicht wirksam.

Resultat ist eine Stadt, die massive Preissteigerungen bei Miet-, noch stärker bei Eigentumswohnungen aufweist. Diese führen dazu, dass große Teile der Bevölkerung einen wachsenden Anteil ihres Einkommens für das Wohnen ausgeben müssen.

Bislang behandelt die Literatur der Wohnungsmarktfoschung entweder den Konnex von Finanzialisierungsprozessen und dem Wohnungsmarkt (zumeist von großen Metropolen) oder den negativen Auswirkungen von Tourismus auf Wohnungsmärkte. Eine gemeinsame und verschränkte Betrachtung dieser beiden Prozesse ist bislang eine Lücke in der aktuellen Stadt- und Wohnungsmarktforschung. Gerade die Verknüpfung der Diskussionen um Finanzanlagen und Tourismus vermittelt unserer Ansicht nach wichtige zusätzliche Erkenntnisse. Beides zusammen macht die regionale Wohnungsfrage und die Preisentwicklung in Städten mit ausgesprägter Nachfrage von außerhalb wie in Tourismus- oder Universitätsstädten erst greifbar und verständlich. Salzburg steht hierbei als Paradebeispiel dafür.

Für Salzburg zeigen wir stellvertretend auf, dass Tourismus und Finanzialisierung getrennte Phänomene auf Wohnungsmärkten begründen, manche Phänomene aber dezidiert auf beide Entwicklungen zurückgeführt werden können.

(Massen-)Tourismus und Finanzialisierung bedingen sich teilweise auch bzw. begünstigen sich wechselseitig. 
Touristische Nachfrage bietet eine Möglichkeit der Renditeerhöhung bzw. -sicherung von Anlagen in Immobilien. Durch den Tourismus gib es zusätzliche Nachfrage nach (Wohn-)Raum. Davon profitieren letztlich immer auch die Eigentümer.

Finanzialisierungsprozesse entfalten sich in Städten mit starkem Tourismus somit auf ihre spezifische Art und Weise.

Grundsätzlich ist die Erzielung von Renten durch Wohneigentum kein neues Phänomen. Doch die Zunahme touristisch genutzter Wohnungen und von Airbnb-Angeboten ist so markant, dass neue Marktsegmente mit ihren eigenen Dynamiken entstanden sind, die auch preissteigernd auf andere Teilmärkte wirken. Konkret in Salzburg ist vor allem in der zentralen Altstadt die gesamte Infrastruktur auf den Tourismus ausgerichtet. Zweitwohnsitze sind ebenfalls vor allem durch den Tourismus geprägte Realität in Salzburg. Fallen Anlageinteressen und touristische Interessen an Salzburg zusammen, sind private genutzte Vorsorgewohnungen das Resultat. Gleichzeitig stehen mehrere tausend Wohnungen leer. Deren Eigentümer_innen halten es für vorteilhafter auf die Wertsteigerung der Immobilie zu setzen, als sie zu vermieten. Werden diese nicht selbst genutzt, werden Sie entweder vermietet, oder einer häufig lukrativeren (touristischen) Kurzzeitvermietung zugeführt. Größere Kapitalanlagemöglichkeiten, die weniger von touristischen Prozessen betroffen sind, stellen Zinshäuser und die Privatisierung öffentlicher Wohnungsbestände dar. Letzteres allerdings in Salzburg nur in geringem Ausmaß.

Die touristische Wohnraumnachfrage bietet ein zusätzliches Anlagefeld mit hoher Dynamik, der durch Finanzanlagen gehaltene Wohnraum ermöglicht touristischer Nutzung zusätzlichen Entfaltungsspielraum. Diese Verbindungskanäle gilt es nicht nur für Salzburg, sondern auch für andere Städte ähnlicher Größe und Struktur noch verstärkt offen zu legen um die regionale Wohnungsfrage politisch beantworten zu können.

Datenverfügbarkeit. Für diesen Artikel wurden keine Datensätze genutzt.

Autorenmitwirkung. Die Autoren trugen zu gleichen Teilen zur grundsätzlichen Konzeption des Artikels bei. AVH ist Hauptautor und trug maßgeblich die Kapitel 1, 3, 4, 5 und 6. CS trug wesentlich zu Kapitel 1, 3 und 5.1 bei, CZ verantwortet mitunter das theoretische Fundament von Kapitel 2, KK leistete Beiträge zu Kapitel 4.

Interessenkonflikt. Die Autoren erklären, dass kein Interessenskonflikt besteht.
Danksagung. Wir stützen unseren Artikel auf verschiedene Vorarbeiten: Working Paper zur generellen Preisentwicklung (Kircher et al., 2018) und zu Wohnsituationen, -zufriedenheit und -kosten in einem Salzburger Stadtteil (Christoforow et al., 2018), auf eine studentische Abschlussarbeit zur Wohnbauförderung (Kautzschmann, unveröffentlichtes Dokument) sowie Sekundärliteratur.

Begutachtung. This paper was edited by Benedikt Korf and reviewed by two anonymous referees.

\section{Literatur}

Aalbers, M. B.: The financialization of housing, Routledge, London, 2016.

Amann, W. and Lugger, K.: Österreichisches Wohnhandbuch 2016, StudienVerlag, Innsbruck, 2016.

Amann, W. and Mundt, A.: Bewertung des Salzburger Modells der Wohnbauförderung, IIBW - Institut für Immobilien, Bauen und Wohnen; im Auftrag der Arbeiterkammer Salzburg, Wien, 2017.

Amann, W., Mundt, A., and Wieser, R.: Berichtsstandard Wohnbauförderung 2014, IIBW - Institut für Immobilien, Bauen und Wohnen GmbH; im Auftrag des Landes Wien, Wien, 2014.

AXA Deutschland: Deutschland-Report 2016, Ruhestandsplanung und -management, Kernergebnisse, online aufrufbar: https://www.axa.de/site/axade/get/documents/axade/AXA. de_Dokumente_und_Bilder/Unternehmen/Presse/Mediathek/ Dokumente/AXA-Deutschland-Report-2016/Kernergebnisse. pdf (letzter Zugriff: 22 Juni 2019), 2016.

Belina, B.: Wenn Geldkapital eine sichere Bank sucht, PROKLA, Z. Krit. Sozialwissen., 48, 187-203, 2018.

Bichler, T.: Wohnbedarfserhebung 2017 für das Bundesland Salzburg, Forum Wohnungslosenhilfe Salzburg, Salzburg, 2018.

Blickhan, M., Bürk, T., and Grube, N.: Touristification in berlin, Sub-urban, s u b-u r b a n Zeitschrift für kritische Stadtforschung, 2, 167-180, 2014.

Chesnais, F.: Finance capital today. Corporations and banks in the lasting global slump, Historical materialism 131, Brill, Leiden, Boston, 2016.

Christoforow, A., Hofmann, M., Majewski, R., Piotrowski, B., Reil, K., Scherer, T., Voggenberger, C., and Vorage, M. W.: Leben und Wohnen in Salzburg-Lehen, Geogr. Uneven Dev., 9, 1-96, https://doi.org/10.25598/goud/2018-9-2, 2018.

CIMA: Einzelhandelsstrukturanalyse Salzburg, CIMA, Salzburg, 2015.

CIMA: Studie zu Besuchermotiven Altstadt Salzburg, CIMA, Salzburg, 2016.

Die Presse: Salzburg. Spontankäufe im gehobenen Millionenbereich, online aufrufbar: https://diepresse. com/home/schaufenster/design/5055733/Salzburg_

Spontankaeufe-im-gehobenen-Millionenbereich (letzter Zugriff: 22 Juni 2019), 2016.

Dopsch, H. and Hoffmann, R.: Salzburg - die Geschichte einer Stadt, Pustet, Salzburg, 2008.

Dörry, S.: Europäische Finanzzentren im Sog der Finanzialisierung, Bundesinstitut für Bau-, Stadt-und Raumforschung (BBSR) im Bundesamt für Bauwesen und Raumordnung (BBR), Internatio- 
nale Immobilienmärkte-globale Immobilienwirtschaft, Informationen zur Raumentwicklung, 5/6, 351-364, 2010.

EHL: Vorsorgewohnungen in Wien Marktbericht 2018, EHL, Wien, 2018.

Engel \& Völkers: Wohnimmobilien. Marktbericht Deutschland 2013/14, Engel \& Völkers, Hamburg, 2013.

Engfer, U.: Ruhestandsmigration und Reurbanisierung, Raumforschung und Raumordnung - Spatial Research and Planning, 76, 35-49, https://doi.org/10.1007/s13147-017-0513-0, 2018.

ERGO: Ergo Risiko-Report 2018, online aufrufbar: https://www.ergo.com/de/Media-Relations/Pressemeldungen/ PM-2018/20180214-ERGO-Risiko-Report (letzter Zugriff: 22 Juni 2019), 2018.

Esping-Andersen, G.: The three worlds of welfare capitalism, John Wiley \& Sons, Cambridge, 2013.

Fehlberg, T. and Mießner, M.: Mietpreissteigerungen und Wohnungsengpässe abseits der Ballungsräume, Investitionen in Wohnimmobilien in B-Lagen und Regionalzentren - das Beispiel Göttingen, s u b-u r b a n Zeitschrift für kritische Stadtforschung, 3, 25-44, 2015

Feichtinger, A. and Schinnagl, M.: Die Vermögensbindung als Eckpfeiler der Wohnungsgemeinnützigkeit, wohnrechtliche blätter: wobl, Wien, 2017.

Fernandez, R. and Aalbers, M. B.: Financialization and housing: Between globalization and varieties of capitalism, Competit. Change, 20, 71-88, 2016.

Freytag, T. and Glatter, J.: Touristification of urban neighborhoods - synergies and conflicts between adequate tourism for the city and the adequate city for tourism, Geogr. Z., 105, 163-166, 2017.

Freytag, T. and Popp, M.: Der Erfolg des europäischen Städtetourismus: Grundlagen, Entwicklungen, Wirkungen, Geogr. Rundschau, 61/2, 4-11, 2009.

Ghose, R.: Big sky or big sprawl? Rural gentrification and the changing cultural landscape of missoula, montana, Urban Geogr., 25, 528-549, https://doi.org/10.2747/0272-3638.25.6.528, 2004.

Gosnell, H. and Abrams, J.: Amenity migration: Diverse conceptualizations of drivers, socioeconomic dimensions, and emerging challenges, GeoJournal, 76, 303-322, https://doi.org/10.1007/s10708-009-9295-4, 2011.

Harvey, D.: The limits to capital, Basil Blackwell, Oxford, 478 pp., 1982.

Harvey, D.: The art of rent: Globalisation, monopoly and the commodification of culture in: Socialist register 2002: A world of contradictions, The Merlin Press, London, 93-110, 2001.

Heeg, S.: Wohnungen als Finanzanlage. Auswirkungen von Responsibilisierung und Finanzialisierung im Bereich des Wohnens, s u b-u r b a n Zeitschrift für kritische Stadtforschung, 1/1, 75-99, 2013.

Helbrecht, I. and Geilenkeuser, T.: Demographischer wandel, Generationeneffekte und Wohnungsmarktentwicklung: Wohneigentum als Altersvorsorge?, Raumforsch. Raumord., 70, 425-436, 2012.

Hennig, C.: Reiselust, Suhrkamp, Berlin, 1999.

Hesse, M. and Preckwinkel, W. J. Z. F. W.: Globalisierung der Wohnungswirtschaft, Z. Wirtschaftsgeogr., 53, 156-171, 2009.

Hoffmann, R. and Luger, K.: Tourismus und sozialer Wandel, in: Geschichte der österreichischen Bundesländer seit 1945. Salzburg, Herausgeber: Dachs, H., Hanisch, E., und Kriechbaumer, R., Böhlau, Wien, 168-209, 1997.
Hölzl \& Hubner: Der Salzburger Immobilienmarkt, Analyse und Immobilien Index SIX, Hölzl \& Hubner, Salzburg, 2018.

Hopfinger, H.: Tourismusatlas Deutschland, Z. Tourismuswissen., 9, 385-386, 2017.

Hudej Zinshäuser: Medienkonferenz am 1. März 2018, Wien, 2018.

IMAS: Lebensqualität \& Lebensstandard - Heute für Morgen. Studie für die Salzburger Sparkasse, online aufrufbar: https://www.sparkasse.at/salzburg/presseaussendungen/2019/ 01/25/IMAS- Vorsorgestudie, letzter Zugriff: 22 Juni 2019.

Jäger, J.: Akkumulation und Wohnungspolitik, Kurswechsel, 3, 3137, 2006.

Jezek, P. C.: Raiffeisen Immobilien erfindet sich neu, Medianet, online aufrufbar: https://medianet.at/news/real-estate/ raiffeisen-immobilien-erfindet-sich-neu-24567.html, letzter $\mathrm{Zu}$ griff: 22 Juni 2019.

Kadi, J., Plank, L., and Seidl, R.: Airbnb: Ein Geschäft für Immobilienprofis, online aufrufbar: https: //awblog.at/airbnb-ein-geschaeft-fuer-immobilienprofis/ (letzter Zugriff: 22 Juni 2019), 2018.

Kircher, A., Schmiedbauer, E., Van-Hametner, A., and Zeller, C.: Regionale Immobilienmärkte unter Druck. Analyse von Immobilienannoncen für Salzburg 2006-2016, Geogr. Uneven Dev., 8, 1-31, 2018.

König, M.: Der Zweitwohnsitz im österreichischen Recht, Linde Verlag, Wien, 2017.

Kunnert, A. and Baumgartner, J.: Instrumente und Wirkungen der österreichischen Wohnungspolitik, WIFO, Wien, 2012.

Land Salzburg: Wohnbauförderung Jahresbericht 2017, Land Salzburg, Salzburg, 2018.

Land Salzburg: Wohnbauförderung Jahresbericht 2018, Land Salzburg, Salzburg, 2019.

Lebuhn, H., Holm, A., Junker, S., and Neitzel, K.: Wohnverhältnisse in Deutschland - eine Analyse der sozialen Lage in 77 großstädten, Berlin, Düsseldorf, 2017.

Marcuse, P. and Madden, D.: In defense of housing: The politics of crisis, Verso Books, London, New York, 2016.

Neuhold, T.: Salzburger Zentrum ist eine Stadt ohne Bewohner, in: Der Standard, Oscar Bronner, Wien, 2018a.

Neuhold, T.: Anrainerproteste gegen Bustourismus in Salzburg, in: Der Standard, Oscar Bronner, Wien, 2018b.

Notariatskammer, Ö.: Presseinformation Immobilien 2016, online aufrufbar: https://www.notar.at/ de/dienstleistungen/immobilien-kauf-und-verkauf/ presseinformation-immobilien-2016/ (letzter Zugriff: $22 \mathrm{Ju}-$ ni 2019), 2016.

Nothegger, B.: Anlageobjekt: Begehrte Zinshäuser, in: Kurier, Helmut Brandstätter, Wien, 2018.

Novy, J. and Colomb, C.: Protest and resistance in the tourist city, Routledge, London, New York, 2017.

OECD: Housing and the economy: Policies for renovation, in: Economic policy reforms 2011. Going for growth, OECD, Paris, 3 24, 2011.

OVI: Pressekonferenz - Marktrück- und ausblick, online aufrufbar: http://www.ovi.at/fileadmin/user_upload/Pressetexte/ Presseunterlagen_Markt_2016_2017.pdf (letzter Zugriff: 22 Juni 2019), 2017.

ÖVI: Övi Marktbericht 2017 - Österreichs Landeshauptstädte im Fokus, ÖVI, Wien, 2018. 
Putschlögl, M.: Vorsorgewohnungen: Steuerreform bringt neue Regeln, online aufrufbar: https://derstandard.at/2000024291610/ Vorsorgewohnungen-Steuerreform-bringt-neue-Regeln (letzter Zugriff: 22 Juni 2019), 2015.

Rechnungshof: Gemeinnützige Salzburger WohnbauGesellschaft mBH, online aufrufbar: http://www.rechnungshof. gv.at/fileadmin/downloads/2007/berichte/teilberichte/salzburg/ Salzburg_2007_08/Salzburg_2007_08_1.pdf (letzter Zugriff: 22 Juni 2019), 2007.

Reutter, G.: Regionale Immobilienmärkte - interessant auch für ausländische Investoren, Immobilien \& Finanzierung - Der Langfristige Kredit, 05/06, 168-169, 2012.

Roland Berger: European city tourism 2015. Study - analysis and findings, online aufrufbar: https://www.rolandberger.com/ de/Publications/European-city-tourism-2015.html (letzter Zugriff: 22 Juni 2019), 2015.

Roland Berger: Protecting your city from overtourism, online aufrufbar: https://www.rolandberger.com/en/Publications/ Overtourism-in-EuropelT1\textbackslash\%27s-cities.html (letzter Zugriff: 22 Juni 2019), 2018.

Romainville, A.: The financialization of housing production in brussels, Int. J. Urban Reg. Res., 41, 623-641, https://doi.org/10.1111/1468-2427.12517, 2017.

Ronald, R. and Kadi, J.: The revival of private landlords in britain's post-homeownership society, New Polit. Econ., 23, 786-803, https://doi.org/10.1080/13563467.2017.1401055, 2017.

Scharmanski, A.: Global denken, lokal handeln: Immobilienwirtschaft im Zeichen der Globalisierung aus theoretischkonzeptioneller Perspektive, Wirtschafts-und Sozialgeograph. Inst., Köln, 2006.

Schoibl, H.: Wohnungslosigkeit und Wohnungslosenhilfe in Salzburg, Helix - Forschung und Beratung, Salzburg, 2011.

Schönig, B., Kadi, J., and Schipper, S.: Wohnraum für alle?!: Perspektiven auf Planung, Politik und Architektur, transcript Verlag, Bielefeld, 2017.

Schorn, J.: Die Altstadt als Altenpsenion und Objekt der Spekulanten, in: Salzburger Nachrichten, Max Dasch, Salzburg, 1992.

Seidl, R., Plank, L., and Kadi, J.: Airbnb in Wien: eine Analyse, interaktiver Forschungsbericht, Wien, online aufrufbar: http: //wherebnb.in/wien (letzter Zugriff: 19 Oktober 2017), 2019.

Seraphin, H., Sheeran, P., and Pilato, M.: Over-tourism and the fall of venice as a destination, J. Destinat. Market. Manage., 9, 374376, 2018.

Serfati, C.: Transnational groups as financial groups, Work Organisat. Labour Globalisat., 5, 10-38, 2011.

Sparkasse Salzburg: Wohnstudie, online aufrufbar: https: //www.sparkasse.at/salzburg/presseaussendungen/2018/06/ 14/Wohnstudie_Salzburg_2018 (letzter Zugriff: 22 Juni 2019), 2018.

Spitzer, W., Reithofer, J., and Prinz, T.: Monitoring der Nachverdichtung in der Stadt Salzburg, AGIT - J. Angw. Geoinform., 3/2017, 383-389, 2017.
Stadt Salzburg: Einwohner der Stadt Salzburg mit Hauptwohnsitz nach Zählbezirk, Altersgruppe, Geschlecht und Staatsbürgerschaft 2008, online aufrufbar: https://www.data.gv.at/katalog/dataset/ogdsbg-einwohnerhauptwohnsitz-zaehlbezirk-altersgruppe- (letzter Zugriff: 22 Juni 2019), 2013.

Stadt Salzburg: Salzburg in Zahlen 3/2018, MA 2/01 Stadtarchiv und Statistik, Stadt Salzburg, Salzburg, 2018a.

Stadt Salzburg: Bevölkerung der Stadt Salzburg nach statistischem Zählsprengel, Wohnsitzart und Geschlecht, Stichtag 1.1.2018, online aufrufbar: https://www.stadt-salzburg.at/pdf/ bevoelkerung_nach_statistischem_zaehlsprengel.pdf (letzter Zugriff: 22 Juni 2019), 2018b.

Stadt Salzburg: Der Tourismus im Jahr 2018, in: Salzburg in Zahlen Stadt Salzburg, Stadt Salzburg, Salzburg, 2019.

Statistik Austria: Wohnsituation in Hauptwohnsitzwohnungen nach Bundesland, online aufrufbar: https://www.statistik.at/web_de/ statistiken/menschen_und_gesellschaft/wohnen/wohnsituation/ 079260.html (letzter Zugriff: 22 Juni 2019), 2018.

Stedman, R. C., Goetz, S. J., and Weagraff, B.: Does second home development adversely affect rural life?, in: Population change and rural society, edited by: Kandel, W. A. and Brown, D. L., Springer Netherlands, Dordrecht, 277-292, 2006.

Straß1, I. and Riedler, W.: Wohnungsleerstand in der Stadt Salzburg, SIR - Salzburger Institut für Raumordnung \& Wohnen, Salzburg, 2015.

Straß1, I., Riedler, W., Uray, R., and Standl, C.: Mietwohnbau in der Stadt Salzburg. Entwicklung und Status, SIR - Salzburger Institut für Raumordnung \& Wohnen, Salzburg, 2018.

Streimelweger, A.: Wohnbauförderung: Eine Bestandsaufnahme, Wirtschaft Gesellschaft, 36, 543-561, 2010.

Van-Hametner, A. and Zeller, C.: Die Anatomie urbaner Immobilienpreisregime, Komparative Analyse von Fundamentalfaktoren und institutionellen Rahmenbedingungen in Linz und Salzburg, Mitteilungen der Österreichischen Geographischen Gesellschaft, 160/2018, 57-90, 2019.

Weichhart, P.: Wohnsitzpräferenzen und „Neue Wohnungsnot“ das Beispiel Salzburg, disP - The Planning Review, 24, 44-51, 1988.

WKO: Immobilienpreisspiegel, WKO, Wien, 2002-2018.

Zeller, C.: From the gene to the globe: Extracting rents based on intellectual property monopolies, Rev. Int. Polit. Econ., 15, 86115, 2008.

Zeller, C., Van-Hametner, A., Smigiel, C., and Kautzschmann, K.: Wohnen in Österreich: Von der sozialen Infrastruktur zur Finanzanlage, PROKLA - Zeitschrift für kritische Sozialwissenschaft, 48, 597-616, https://doi.org/10.32387/prokla.v48i193.1148, 2018. 